\title{
The Causal Relationships between Inflation and Inflation Uncertainty
}

\author{
William A. Barnett \\ University of Kansas, Lawrence, and Center for Financial Stability, NY City \\ Zied Ftiti \\ EDC Paris Business School, France \\ Fredj Jawadi \\ University of Evry, France
}

April 8, 2018

\begin{abstract}
Since the publication of Friedman's (1977) Nobel lecture, the relationship between the mean function of the inflation stochastic process and its uncertainty has been the subject of much research. Friedman postulated that high inflation causes increased inflation uncertainty. Ball (1992) produces macroeconomic theory that could justify that causality. But other researchers have found the converse causality, from increased inflation uncertainty to increased mean inflation, and postulated macroeconomic theory that could support their views. In addition, some researchers have found inverse correlation between mean inflation and inflation volatility with causation in either direction. These controversies are important, since they have different implications for economic theory and policy. We conduct a systematic econometric study of the relationship among the first two moments of the inflation stochastic process using state of the art approaches.
\end{abstract}

We propose a time-varying inflation uncertainty measure based on stochastic volatility to take into account unpredictable shocks. Further, we extend previous related literature by providing a new econometric specification of this relationship using two semi-parametric approaches: the frequency evolutionary co-spectral approach and the continuous wavelet methodology. We theoretically justify their use through an extension of Ball's (1992) model. These frequency approaches have two advantages: they provide the analyses for different frequency horizons and do not impose restriction on the data. While related literature always focused on the US data, our study explores this relationship for five major developed and emerging countries (the US, the UK, the Euro area, South Africa, and China) over the last five decades to investigate robustness of our inferences and investigate sources of prior inconsistencies in inferences among prior studies. This selection of countries permits investigation of the inflation versus inflation uncertainty relationship under different hypotheses, including explicit versus implicit inflation targets, conventional versus unconventional monetary policy, independent versus dependent central banks, and calm versus crisis periods.

Our findings depict a significant relationship between inflation and inflation uncertainty that varies with time and frequency and offer an improved comprehension of the ambiguous inflation versus inflation uncertainty relationship. This relationship seems positive in the short and medium terms during stable periods, confirming the Friedman-Ball theory, while it is negative during crisis periods. In addition, our analysis identifies the phases of leading and lagging inflation uncertainty. Our general approach nests within it the earlier approaches, permitting explanation of the prior appearances of ambiguity in the relationship and identifies the conditions associated with the various outcomes.

Keywords: Inflation, Inflation uncertainty, Frequency approach, Wavelet, Semi-parametric approach, Stochastic volatility.

JEL: C14, E31. 


\section{Introduction}

Uncertainty, which often refers to unpredictable volatility (Grier and Perry, 1998), is an important concept in economic theory, as it could affect consumers' saving, investors' and policymakers' decisions, economic well-being, and the entire economy (Rossi et al., 2016).

In particular, uncertainty about future inflation, which is considered among the most important inflation costs, is one of the main concerns of monetary policymakers. According to Cukierman and Meltzer (1986) and Evans and Wachtel (1993), inflation uncertainty (IU) can occur through at least two main sources. First, significant differences among international monetary policy regimes could lead to IU, as through conventional versus unconventional monetary policies. Second, IU could also be induced by policy regime uncertainty. ${ }^{1}$ Furthermore, as economic agents often use new information to attempt to update their perceptions regarding the actions of central banks, it is expected that IU would be time varying and potentially complex to measure.

The relationship between inflation and IU has been the focus of several theoretical and empirical studies in order to investigate the latter's effect. Friedman (1977) showed a positive relationship between inflation and IU while suggesting that inflation causes IU. Using a game with asymmetric information and two policymakers, consisting of a liberal policymaker who is prepared to disinflate and bear the economic cost of reducing the inflation and a conservative policymaker who is not prepared to do so, Ball (1992) developed a formal justification for Friedman's theory. This relationship is known as the Friedman-Ball relationship or theory. ${ }^{2}$

\footnotetext{
${ }^{1}$ Policymakers could use information unavailable to the public or could create an inflation surprise, but the public might not know the weight assigned to this surprise creation. Furthermore, policymakers can sometimes find ambiguous procedure to be useful.

2 The principle is that when inflation is low (as was in the US in the 60s), there is a consensus that policymakers will strive to keep it low. However, if the inflation is high (as in the late 70s), there will be a dilemma for policymakers. Either they dis-inflate, which can produce a recession risk, or they do not. The public then will be unclear about intentions of future policymakers. Even if disinflation does occur, its timing would be uncertain, raising IU anyway.
} 
This suggested positive relationship between inflation and IU can be found in Logue and Willet (1976) and Fisher and Modigliani (1978). However, recent related empirical studies, focused on US inflation, indicated some ambiguity in the relationship between inflation and IU. See, e.g., Golub, 1994.

The objectives of our research are analogous to those of Barnett, Gallant, Hinich, Jungeilges, Kaplan, and Jensen (1997), who investigated the source of nonrobustness in time series inferences about nonlinearity, and of Barnett and Chen (2015), who investigated nonrobustness of policy inferences from structural macroeconometric models. But the approaches to resolving the nonrobustness problems differ. Barnett et al (1997) ran a controlled competition among competing time series tests, while Barnett and Chen (2015) investigated bifurcation stratification of the structural parameter spaces. In the current paper, we extend inflation-IU testing to time-frequency analysis to nest prior approaches into our more general econometric analysis.

This study revisits the relationship between inflation and IU to investigate that ambiguity for some developed and emerging countries. We conduct that investigation in a systematic econometric manner using state of the art methodology. The importance of that relationship has been motivated in macroeconomics in many ways. First, the co-existence of targeted and nontargeted monetary policies yields heterogeneous effects on price stability, inflation policy, and subsequently on IU. This can be illustrated by the variety in levels and volatility of inflation among the countries. Second, the fact that some central banks are more independent and have larger mandates than others has many implications for inflation and the relationship between inflation and IU. The more independent is the central bank, the more we might expect an increase in uncertainty to imply an inflation fall, and vice versa. Third, the recent global financial crisis has directly affected liquidity, and prompted some monetary authorities to switch from conventional to unconventional monetary policies, which could increase 
uncertainty and could influence how IU impacts inflation ${ }^{3}$. Fourth, the differences in monetary policy mandates produce different time horizons across countries to achieve the monetary policy goals.

Those horizons can be characterized as short-term (less than one year), medium-term (from one to three years), and long-term (more than three years) . Consequently, we suggest that $a$ priori the relationship between inflation and IU could vary across horizons. We address this concern by using an evolutionary econometric analysis to assess the time-varying effect of IU. This is a central focus of our analysis.

Our study makes two fundamental contributions to this field. We propose a time-varying estimate of IU using the stochastic volatility model of Berument et al. (2009) and Ferreira and Palma (2016) to take unexpected shocks to inflation into account. Unlike previous studies that often apply parametric econometric models, our study applies two non-parametric approaches, the evolutionary co-spectral approach and the wavelet methodology, to reproduce the timevarying relationship between inflation and IU. This new specification of the inflation and IU relationship with non-parametric models can be theoretically justified through our extension of Ball's (1992) model.

Our methodological choice has many advantages. ${ }^{4}$ First, nonparametric approaches do not require restrictions on or pre-treatment of the data, as required for the time-series models. Second, these approaches enable us to investigate this relationship in continuous time to yield a time-varying analysis of the inflation-IU relationship and a decomposition of this relationship

\footnotetext{
${ }^{3}$ Mallick and Mohsin (2016) show that that this switching induces inflation shocks that might affect the real economy and in particular the consumption of durable rather than non-durable goods.

${ }^{4}$ To our knowledge, this is the first study that assesses the relationship between inflation and a time-varying measure of IU using multivariate frequency approaches. In the related literature, two previous works have analyzed inflation and IU relationship through wavelet approach, but using a constant measure of IU (Bouoiyour and Selmi, 2014; Albulescu et al. 2015), which can bias results. Bouoiyour and Selmi (2014) have used the maximal overlap discrete wavelet transformations (MODWT), but with several strong assumptions.
} 
across different horizons. Third, the wavelet approach transforms these time series into different frequency components, thus providing an alternative representation of the variability between variables on a scale-by-scale basis. The result is a more informative analysis of the inflationIU relationship, while identifying, for example, the periods of lead and lag uncertainty. Our results are particularly productive in resolving the ambiguity associated with this relationship, as discussed by Golub (1993).

We focus on a heterogeneous sample of five major developed and emerging countries, including the US, the UK, the Euro area, China, and South Africa. Our objective is to produce conclusions that are robust across difference circumstances. These choices include countries with an explicit inflation target and others with an implicit one. The central banks' commitments vary across countries, enabling us to test the effect of central bank independence on the way uncertainty affects inflation or vice versa. Some of these countries still rely on conventional monetary policies (e.g., China), while others use unconventional monetary policies. This study is the first to focus on the effect of the latter on the inflation-IU relationship. The extension of the sample to the aftermath of the global financial crisis enables us to check this relationship during both calm and crisis periods. The sample period varies by country according to the data availability; but for all countries, we include the data both before and after the recent global financial crisis.

Overall, our sample enables us to test the effects of different hypotheses about inflation and uncertainty. As in the aftermath of the recent global financial crisis, there has been a tendency towards deflation in several countries, while uncertainty remains relevant. This sample should help answer the questions on whether the inflation-IU relationship has changed over the last few years.

Our analysis provides three primary results. First, we note a significant relationship between inflation and IU, varying with time and frequency. More precisely, this relationship alternates 
between being positive in the short and medium terms during stable periods, confirming the Friedman-Ball theory, while becoming negative in crisis periods. Our results distinguish periods where IU is leading from those where it is lagging. This relationship also varies per country, suggesting a significant effect of the monetary policy regime, the target objective, and the degree of central bank independence on uncertainty. Overall, the double specification of this relationship through time and frequency and our theoretical justification provide new findings that could be helpful to predict the uncertainty effects and drivers more reliably. Also our results cast light on the ambiguity associated with this relationship, as found in previous studies (e.g., Golub, 1993).

The remainder of this paper is organized as follows. Section 2 briefly discusses the theoretical framework and the related literature on the inflation-IU relationship. Section 3 presents the econometric methodology. The main empirical results are discussed in Section 4. Section 5 concludes the paper.

\section{Theory and Related Literature}

\subsection{Theoretical Background}

The relationship between inflation and IU has been investigated in the literature in both directions, with each direction drawing upon different economic backgrounds. Table 1 shows that each background is itself divided into two strands, based on the sign of the relationship between inflation and IU.

The first economic background stipulates that inflation causes IU and is disaggregated into two strands based on the sign of this causality direction relationship. Friedman (1977) argued that the relationship between inflation and IU is positive. Indeed, in that Nobel Prize lecture, entitled "Inflation and Unemployment," he stated that the monetary policy objective of boosting employment would raise inflation. In theory, the central bank aims to confront the inflation pressure, but in practice Friedman explained that monetary authorities could behave 
in different ways. Consequently, the public would be uncertain about the future policy, leading to more IU.

In the same context, Ball (1992) provides a more formal justification of Friedman's hypothesis. He uses an asymmetric information game between the Federal Reserve and the public. The game postulates two kinds of policymakers: liberal and conservative. The public knows that a conservative policymaker is willing to bear the economic costs of reducing inflation, while the liberal policymaker is not. The public is not certain about who will be the future policymaker. If the inflation is low, uncertainty will be low as well, since either policymaker will keep the inflation low. However, during high inflation, only the conservative policymaker will disinflate. Thus, when inflation is high, the doubt about the identity of the future policymaker will cause IU also to be high. Based on Ball's model, Friedman's hypothesis would be correct. Increased inflation would raise IU.

Pourgerami and Maskus (1987) also agreed with Friedman's hypothesis on the direction of the relationship between inflation and IU, but they argued that the sign of the relationship is negative. Under a high inflation rate, agents in their model tend to forecast the inflation rate more accurately, since they invest more resources to avoid forecasting errors, having significant related costs. Accordingly, a high inflation rate will decrease the IU.

Table 1: Inflation-IU Relationship Theories

\begin{tabular}{||c|c|c|}
\hline $\begin{array}{c}\text { Sign of the } \\
\text { relationship }\end{array}$ & $(+)$ & $(-)$ \\
\hline Causality of the relationship & & $\begin{array}{c}\text { Pourgerami and Maskus } \\
(1987) \\
\text { Ungar and Zilberfarb } \\
(1993)\end{array}$ \\
\hline Inflation causes IU & Ball (1992) \\
\hline IU causes inflation & Cukierman and Meltzer (1986) & Holland (1995) \\
\hline
\end{tabular}

The second economic background claims that the causality relationship is inverse, with IU 
causing inflation. In this tradition, there also are two contradictory analyses, differing in the sign of the causality relationship from uncertainty to inflation. Cukierman and Meltzer (1986) applied a game theoretic model on central banks. Their framework is based on the BarroGordon model of Federal Reserve behavior, emphasizing money supply uncertainty and the objective function of policymakers. The model concludes that an increase in uncertainty implies a corresponding increase in inflation, as the policymakers seek to create an inflation surprise to stimulate output growth. Then uncertainty positively causes inflation. But conversely, Holland (1995) found that uncertainty can have a negative impact on inflation. The independent central bank decreases inflation following an IU increase, to reduce the real costs of IU.

Overall, it is clear that this relationship between inflation and IU has been the subject of complexity and ambiguity in either direction. ${ }^{5}$ In order to resolve this ambiguity and understand the specificities of the linkages between inflation and IU, we now reconsider the first background framework related to this relationship, the hypothesis stating that inflation causes IU. In particular, we extend Ball's (1992) model to better characterize the shock properties in his model. The resulting extended model motivates our time-frequency analysis of this relationship in a manner that can explain and resolve its ambiguous character.

More formally stated, Ball's (1992) model identifies two types of policymakers. Conservative policymakers (C), who focus only on inflation, and liberal policymakers (L), who focus on unemployment as well as inflation. Their loss functions in period $t$ are represented by Equations (1):

$$
\left\{\begin{array}{c}
L_{t}^{C}=a \pi_{t}^{2} \\
L_{t}^{L}=a \pi_{t}^{2}+\left(U_{t}-U_{t}^{*}\right),
\end{array}\right.
$$

where $L_{t}^{C}$ and $L_{t}^{L}$ are the loss functions for the conservative and liberal policymakers, respectively; $U_{t}$ and $U_{t}^{*}$ represent the actual unemployment and optimal social unemployment,

\footnotetext{
${ }^{5}$ On the subject of complex dynamics, see Barnett, Serletis, and Serletis (2015).
} 
respectively; and $\pi_{t}$ represents the inflation rate at period $t$. The optimal social unemployment is assumed to be time-invariant.

According to the short-run Phillips curve, unemployment is determined as follows:

$$
U_{t}=U^{N}-\left(\pi_{t}-\pi_{t}^{e}\right)
$$

where $\pi_{t}^{e}$ is the expected inflation rate at time $t$, given the available information at period $t$ - 1 . The natural rate of unemployment is $U^{N}$.

In order to avoid the time inconsistency problem, the natural unemployment rate is defined as $U^{N}=U^{*}+1 .^{6}$ Therefore, by combining (1) and (2), the liberal policymaker's loss function is written as follows:

$$
L_{t}^{L}=a \pi_{t}^{2}+\left(\pi_{t}-\pi_{t}^{e}-1\right)
$$

In this model, policymakers are assumed to alternate their power stochastically, based on a Markov process. More specifically, Ball (1992) assumes that in period $t$, L are in power. They are characterized by a probability $p$, at which they would lose the power and would be replaced by $\mathrm{C}$ in the following period $t+1$, and vice versa.

Further, from Canzoneri (1985) and Ball (1992), it is assumed that the policymakers in power define their objective through the following inflation target $\left(\pi_{t}^{*}\right)$ :

$$
\pi_{t}=\pi_{t}^{*}+\delta_{t}
$$

where $\delta_{t}$ presents a stochastic shock.

Equation (4) stipulates that the two policymakers separately define an expected inflation rate $\left(\pi_{t}^{e}\right)$, which is assumed to be rational. Then, the policymakers would individually define their inflation targets $\left(\pi_{t}^{*}\right)$, which depend on the inflation level in the previous period, $t-1$.

In practice, the equilibrium of the policymakers is defined after fixing the inflation target by minimizing the expected present value of their respective loss functions. That is, based on

\footnotetext{
${ }^{6}$ In line with previous studies (Barro and Gordon, 1983), time inconsistency is defined to occur, when the natural rate of unemployment is greater than the optimal social unemployment, leading to an inflation bias.
} 
this game, we have several cases that differ regarding the policymaker in power and the function of the inflation level at $t-1$. These cases are summarized in the following table.

\begin{tabular}{|c|c|}
\hline $\begin{array}{l}\text { Case 1: C are in power } \\
\text { at } t-1\end{array}$ & Case 2: L a \\
\hline $\begin{array}{l}\text { If } C \text { are in power at } \\
t \text { - } 1 \text {, their inflation } \\
\text { expectation for period } t \\
\text { will be zero, as their } \\
\text { monetary objective is } \\
\text { only to reduce inflation. } \\
\text { In addition, the liberal } \\
\text { policymakers expect } \\
\text { zero inflation for period } \\
t \text {, since at } t \text { - } 1 \text { the } \\
\text { conservative } \\
\text { policymakers are in } \\
\text { power. }\end{array}$ & $\begin{array}{l}\text { If } \mathrm{L} \text { are in power at } t \text { - } 1 \text {, their inflation expectation for period } t \text { depends } \\
\text { on the previous inflation level. The liberal policymakers are tempted by a } \\
\text { positive inflation in order to increase employment. However, their } \\
\text { temptation is deterred by their adverse attitude to inflation bias resulting } \\
\text { from temporal inconstancy. Therefore, to avoid such problems, Ball } \\
\text { (1992) supposed that liberal policymakers will be tempted toward a } \\
\text { positive inflation, but less than a threshold level ( } \bar{\pi} \text { ) } \\
\text { Case 2.1: If } \pi_{t-1}<\bar{\pi} \text { (low inflation): } \\
\text { In this case, the liberal policymakers target an inflation rate equal to zero. } \\
\text { This behavior is explained by the fact that } \mathrm{L} \text { are tempted to raise their } \\
\text { target but fear to exceed the threshold } \bar{\pi} \text {. In the case of C, they continue to } \\
\text { target zero inflation. } \\
\text { Case 2.2: If } \pi_{t-1}>\bar{\pi} \text { (high inflation): } \\
\text { In this case, } L \text { target an inflation rate } \pi_{t}^{*+}>\bar{\pi} \text {. Behind their preference, } \mathrm{L} \\
\text { avoid an aggressive reaction to reduce inflation, as they fear recession. } \\
\text { However, conservative policymakers target an inflation rate equal to zero, } \\
\text { as their main objective is to reduce inflation. } \\
\text { As the probability of liberal policymakers still in power is } 1 \text { - p, the } \\
\text { expected inflation will be ( } 1-\text { p) } \pi_{t}^{*+} \text {. }\end{array}$ \\
\hline
\end{tabular}

Based on the above model, the arrival of economic shocks will define the level of inflation at period $t$ (Equation 4). According to all the above cases, the expected inflation rate could take two possible values: zero or $(1-\mathrm{p}) \pi_{t}^{*+}$. When $\pi_{t}^{e}=E\left[\pi_{t} \mid \omega_{t-1}\right]=0$, there is no uncertainty. However, when $\pi_{t}^{e}=E\left[\pi_{t} \mid \omega_{t-1}\right]=(1-\mathrm{p}) \pi_{t}^{*+}$, based on Equation 4 the inflation level at period $t$ will have a variance as follows: 


$$
\operatorname{Var}\left[\pi_{t}\right]=\operatorname{Var}\left[\pi_{t}^{*}+\delta_{t}\right]=\operatorname{Var}\left[\delta_{t}\right]
$$

Equations 1 - 5 summarize Ball's model. To motivate our use of time-scale approaches, which can explore the relationship between inflation both over time and over frequency, we next extend Ball's model by decomposing the shock in Equation (4) into two shocks, as follows:

$$
\delta_{t}=e_{t}^{L}+e_{t}^{S}
$$

where $e_{t}^{L}$ represents a long-term shock, associated with a further change in monetary policy, that might affect the inflation in the long-term (Evans, 1991), while $e_{t}^{s}$ is a short-term shock in money demand, affecting the inflation in the short term (Ball, 1992) ${ }^{7}$.

We suppose that $e_{t}^{L}$ and $e_{t}^{S}$ are serially uncorrelated, independent, and normally distributed with a mean equal to zero, and with variance $\sigma_{L}^{2}$ and $\sigma_{s}^{2}$, respectively. This extension of Ball's model enables the inflation to be differently shocked (short-term versus long-term shock), affecting the actual level of inflation.

Let us now reconsider Equation (5) of the inflation variance, while considering our shock decomposition in Equation (6). Accordingly, the inflation uncertainty, $\operatorname{Var}\left[\pi_{t}\right]$, will depend on the nature of the shocks, short-term versus long-term shock. If there is only a short-term shock and the monetary policy is unchanged, then the inflation uncertainty could be specified as

$$
\operatorname{Var}\left[\pi_{t}\right]=\operatorname{Var}\left[\pi_{t}^{*}+\delta_{t}\right]=\operatorname{Var}\left[\delta_{t}\right]=\operatorname{var}\left[e_{t}^{s}\right]=\sigma_{s}^{2}+p(1-p) \pi_{t}^{*+}
$$

However, if there is a long-term shock, which affects the economy, then the inflation uncertainty could be captured as follows.

$$
\operatorname{Var}\left[\pi_{t}\right]=\operatorname{Var}\left[\pi_{t}^{*}+\delta_{t}\right]=\operatorname{Var}\left[\delta_{t}\right]=\operatorname{var}\left[e_{t}^{L}\right]=\sigma_{L}^{2}+p(1-p) \pi_{t}^{*+}
$$

The above reconsideration of inflation uncertainty dynamics in Ball's (1992) model, through the hypothesis on the shock nature, suggests that inflation uncertainty dynamics might differ,

\footnotetext{
${ }^{7}$ Hereafter, we can empirically show that the interest of using the wavelet and frequency approaches to apprehend the inflation-IU relationship for different time-scales and frequencies can be justified by the presence of different short and long-term shocks.
} 
and exhibits further asymmetry and complexity, according to the type and horizon of the shock affecting the economy. In addition, IU's interaction with inflation might differ with the horizon and the type of the inflation shock. Thus, for a better characterization of this inflation-IU relationship, we investigate it for different time horizons and scales by appealing to a timefrequency approach. This empirical approach will be discussed further after reviewing the related literature.

\subsection{Related Literature}

Several recent theoretical and empirical studies have focused on the inflation-IU relationship, but there continues to be no consensus about either the direction or the sign of this relationship. ${ }^{8}$ Using the autoregressive conditional heteroscedasticity (ARCH) and Generalized ARCH (GARCH models), Ben Nasr et al. (2015) concluded that the average US inflation is not related to uncertainty. Baillie et al. (1996) applied a fractionally integrated GARCH model and also found no relationship between inflation and uncertainty in the US. However, they found significant linkages in the UK, Brazil, Argentina, and Israel. Grier and Perry (1998) investigated the inflation-IU relationship for the G7 countries during 1948-1993. While their causality analysis supports the Friedman-Ball hypothesis that inflation Granger causes uncertainty, there are mixed results regarding the causality effect from uncertainty to inflation. Their results vary among countries, and the authors have mentioned that the response to IU might be correlated with measures of central bank independence. Indeed, for Japan and France, the relationship obtained is consistent with Cukierman and Meltzer (1986), in which increased uncertainty is related to higher inflation, while for the US, Germany, and the UK, the result is the opposite.

Kontonikas (2005) studied the relationship between IU and inflation in the UK during 1972-2002 and found a positive correlation between past inflation and current uncertainty. In

\footnotetext{
${ }^{8}$ We intentionally mention only the recent, important, and related studies; see Ben Nasr et al. (2015) for a more complete literature review. Golob (1993) also provides a concise analysis and a survey on this complex relationship.
} 
addition, the author showed that adopting an explicit inflation target by the Bank of England reduces inflation persistence and uncertainty. Applying parametric models of long memory to the US, the UK, and Japan during 1962-2001, Conrad and Karanasos (2005) validated the Friedman hypothesis that inflation raises IU. Furthermore, they found that IU affects inflation in Japan and the UK differently. Using a stochastic volatility in mean model, Berument et al. (2009) studied the effect of IU on inflation in the US during 1976-2006. The authors found an increase in inflation following a positive shock affecting inflation volatility. That result is in line with Cukierman and Meltzer (1986) and Cukierman (1992).

Neanidis and Savva (2011) investigated the relationship between nominal uncertainty and inflation in the context of the European Union (EU) and found that uncertainty positively affects inflation in the pre-EU access period. Further, they indicated the absence of any effect during EU access and entry. Using a Markov-regime switching asymmetric GARCH-in-mean model, Chang (2012) investigated the inflation-IU relationship under the hypotheses of regime switching and non-normality in the US during 1960-2011. The author showed that IU does not affect inflation, while the inflation affects IU negatively in periods of high-inflation volatility, but not in periods of low-inflation volatility. The main advantage of the research of Chang (2012) is in relaxing the restrictions on the distribution of random errors and the paper's use of a switching-regime framework. Zapodeanu et al. (2014), focused on Romania, showed a significant bilateral-causality relationship between inflation and IU. Mallick and Sousa (2013) focus on the BRICS and found that important commodity price shocks might lead to a rise in inflation and require an aggressive action from central banks towards inflation stabilization, yielding inflation uncertainty.

Finally, Ben Nasr et al. (2015) also applied the Markov-switching vector autoregressive model to investigate the relationship between inflation and IU in South Africa during 19212012. Their findings did not reject Friedman's hypothesis, but the causality relationship was 
found to be valid only if unidirectional. Creal and Wu (2014) developed a new macro-financial model to study the effect of interest rate uncertainty on business cycles. They showed that a shock to the short interest rate negatively affects inflation while a higher long-term uncertainty shock has a positive effect on it. A related literature investigates the relationship between money supply growth uncertainty and the economy. See, e.g., Serletis and Rahman (2009a,b), Serletis and Shahmoradi (2006), and Serletis and Xu (2017).

Overall, previous studies do not provide a unanimous conclusion about the inflation-IU relationship, and their findings vary with the implemented methodologies, countries, and samples under consideration. However, all previous studies tested this relationship using parametric models, which imply restrictions on the specification of the relationship between inflation and uncertainty. In addition, it seems a priori that the sign and size of the IU effect on inflation depends on the level of the central bank's commitment to inflation. Indeed, with a weak commitment, a positive effect can be expected, while a negative effect is anticipated if the central bank has a strong commitment. Also, it appears that the effect of inflation on uncertainty might differ depending on the conduct of explicit or implicit inflation targets. Johnson (2002) showed that while a formal target has a negative effect on inflation, it significantly impacts uncertainty.

All these different empirical findings confirm a related study by Golub (1993), who showed that both survey strategy, based on surveys of economists and consumers, and forecasting model strategy, based on Restricted-Uncertainty (GARCH) Models of IU across exchange rate regimes, yield mixed findings and ambiguity when considering the inflation-IU relationship. ${ }^{9}$ To reconcile this disagreement, he considered the hypothesis of a downtrend in uncertainty and showed that the non-consideration of such effects in previous studies may have biased the

\footnotetext{
${ }^{9}$ In particular, for Golub (1993), survey analyses confirm the Friedman-Ball hypothesis, while the forecasting approaches are less conclusive as restricted-uncertainty models provide mixed results, and the exchange rate model found no relationship at all between inflation and IU.
} 
previous results.

In our paper, we propose to resolve this ambiguity and the inconsistent results in previous research on the inflation-IU relationship, while proposing an alternative, more general strategy. Contrary to the dominate strand of literature using variants of the GARCH model in modelling IU, we present a time-varying latent measure of IU based on stochastic volatility, in line with Berument et al (2009), Chan and Grant (2016), and Ftiti and Jawadi (2018). Moreover, we adopt a time-frequency approach that nests prior approaches within our more general approach.

\section{Econometric Methodology}

\subsection{Measure of Inflation Uncertainty}

Before moving to the analysis of the relationship between inflation and IU, we need to provide a measure for IU. The earlier related literature used the standard deviation or the variance of inflation as proxies for IU. However, these measures have been criticized as capturing only inflation variability and not IU, which depends upon variations of that nonconstant variance. Subsequently, ARCH and GARCH models have used the conditional variance of inflation as a proxy for IU (e.g., Emery, 1993; Holland, 1993). Although studies, such as Kontonikas (2005) and Ben Nasr (2011), have employed variants of GARCH models as a proxy of IU, recently they also have been criticized.

Therefore, other studies (Berument et al, 2009; Ferreira and Palma, 2016) modeled the conditional variance as an unobserved component based on a Markov process, known as stochastic volatility (SV) models. In addition to the time-varying behavior of the SV models, they are considered as more flexible than previous classical measures, as SV models embody two separate disturbance terms, (Carnero et al., 2004). Furthermore, the latent specification in the SV models rejects any ad-hoc assumptions on the specification of the conditional volatility. In addition, The SV proxy is more appropriate to capture the unpredictable characteristics of uncertainty 
In practice, Chan and Grant (2016) have recently compared different variants of the GARCH model and different SV models and confirmed the superiority of the latter. Although this kind of measure is mostly adopted for financial time series analysis (Gourieroux and Sufana, 2010; Koopman et al., 2010), some recent papers developed stochastic volatility measures for economic time series, such as inflation (Chan, 2015) and exchange rate (Chan and Hsiao, 2014). More interestingly, in the literature on inflation and IU, the SV model has been used as a measure of IU in Berument et al. (2009), Chan (2015), Ferreira et Palma (2016), and Ftiti and Jawadi (2018), but with heterogeneous results.

In this study, we retain the stochastic volatility as a proxy of IU. Specifically, we apply a stochastic volatility model based on moving average student-t-errors, developed by Chan (2013). ${ }^{10}$ To estimate the stochastic IU, we adopt the efficient sampler method proposed in Chan (2013), specified as follows.

The inflation series is represented as follows:

$$
\begin{aligned}
& \pi_{t}=\mu+\tau_{t}, \\
& \tau_{t}=\epsilon_{t}+\psi \epsilon_{t-1},
\end{aligned}
$$

where $\epsilon_{t} \sim N\left(0, \exp \left(h_{t}\right)\right)$ for $t=1, \ldots, T$. However, the state is assumed to evolve into a stationary condition based on the following equation:

$$
h_{t}=\mu_{h}+\phi_{h}\left(h_{t-1}-\mu_{h}\right)+\zeta_{t}
$$

where $\zeta_{t} \sim N\left(0, \sigma_{h}^{2}\right)$ for $t=1, \ldots, T$, with $\zeta_{t}$ and $\epsilon_{t}$ independent for all leads and lags. The stationarity condition of $\left(h_{t}\right)$ is $\left|\phi_{h}\right|<1$. The states are initialized with $h_{1} \sim N\left(\mu_{h}, \frac{\sigma_{h}^{2}}{1-\phi_{h}^{2}}\right)$.

This specification is completed with independent prior distributions for $\mu_{h}, \phi_{h}$, and $\sigma_{h}^{2}$, such that

\footnotetext{
${ }^{10}$ There are also other methods of modeling of stochastic volatility less interesting such as Gaussian errors models or heavy tails and serial dependence.
} 


$$
\mu_{h} \sim N\left(\mu_{h_{0}}, V_{\mu_{h}}\right) ; \quad \phi_{h} \sim N\left(\phi_{h_{0}}, V_{\phi_{h}}\right) I\left(\left|\phi_{h}\right|<1\right) ; \text { and } \sigma_{h}^{2} \sim I G\left(v_{h}, S_{h}\right),
$$

where $I[*]$ is an indicator function and IG is the inverse-gamma distribution.

\subsection{Time-Scale Approaches}

Unlike previous studies, we rely on two time-scale approaches to investigate the relationship between inflation and IU: the evolutionary co-spectral approach and the wavelet method. These approaches have at least two advantages over the well-known time-series models. First, they provide analysis at multiple frequencies. Consequently, the analysis of this relationship is explored in the short, medium, and long terms. Second, these approaches are non-parametric. Therefore, they require no hypotheses about the distributions and no estimation of parameters.

\subsubsection{Evolutionary Co-spectral Density Function}

Priestley (1965) extended the spectral approach for stationary processes to the nonstationary case by proposing the evolutionary spectral approach. The word "evolutionary" refers to a time-dependent time series $X(t)$. This approach has been used recently by several authors, such as Allégret and Essaadi (2011); Ftiti (2010); Van Bellegem (2013); and Van Bellegem and Von Sachs (2008). Priestley and Tong (1973) extended the analysis to the bivariate case, which will be useful to study the relationship between two processes associated with the inflation series, based on the coherence measure $C_{t, X Y}^{2}(w)$. We denote those two series by $X(t)$, which is inflation, and $Y(t)$, which will be IU. The coherence function is interpreted as a linear relationship between the corresponding components of the studied time series $X(t)$ and $Y(t)$. We note that this measure is equivalent to the correlation in the time-domain approach, except that the signal is squared in the case of our coherence measure. Therefore, the coherence measure ranges from zero to one, while the classical correlation ranges from -1 to 1 .

Coherence is defined as follows: 


$$
C_{t, X Y}^{2}(w)=\frac{h^{2}{ }_{t, X Y}}{h_{t, X X} h_{t, Y Y}},
$$

where $h_{t, X X}$ and $h_{t, Y Y}$ are the estimated auto-spectral density function for inflation, $X(t)$, and inflation uncertainty, $Y(t)$. The cross-spectral density function between the two processes is $h_{t, X Y} \cdot{ }^{11}$

Priestley $(1965,1966)$ defined the suitable windows to make robust estimation for the spectral and co-spectral density functions as:

$$
g(u)=\left\{\begin{array}{ll}
\frac{1}{2 \sqrt{h \pi}} & \text { if }|u| \preccurlyeq h \\
0 & \text { if }|u|>h
\end{array} \quad W_{v}= \begin{cases}\frac{1}{T^{\prime}} & \text { if }|v| \preccurlyeq \frac{T^{\prime}}{2} \\
0 & \text { if }|v|>\frac{T^{\prime}}{2}\end{cases}\right.
$$

In accordance with Priestly’s definition, the symbol $\preccurlyeq$ means less than or equal to, but close to, and with small probability possibly greater than. We adopt the same window parameters used by Artis et al. (1992), $h=7$ and $T^{\prime}=20$. This choice of values is consistent with the conditions (i) and (ii) below and provides robust estimators. With $\hat{\mathrm{h}}_{k}(t, w)$ being the estimate of the spectral density, $h_{k}(t, w)$, Priestley (1988) concluded that $\hat{\mathrm{h}}_{k}(t, w) \approx h_{k}(t, w)$, while var $\hat{\mathrm{h}}_{k}(t, w)$ decreases, when $T^{\prime}$ increases, and $\operatorname{cov}\left(\hat{h}\left(t_{1}, \mathrm{w}_{1}\right), \hat{h}\left(t_{2}, \mathrm{w}_{2}\right)\right)=0, \quad \forall\left(t_{1}, t_{2}\right)$, $\forall\left(w_{1}, w_{2}\right)$, if at least one of the following conditions is satisfied: $(i)\left|t_{1}-t_{2}\right| \geq T^{\prime}$ and (ii) $\left|w_{1} \pm w_{2}\right| \geq \frac{\pi}{h}$. To respect conditions ( $i$ ) and (ii), we choose $\left\{t_{i}\right\}$ and $\left\{w_{j}\right\}$ so that:

$$
t_{i}=\{18+20 i\}_{i=1}^{I},
$$

where $I=\left[\frac{T}{20}\right]$ with $T$ being the sample size, and

$$
w_{j}=\left\{\frac{\pi}{20}(1+3(j-1)\}_{j=1}^{7}\right.
$$

To consider condition (ii), the following frequencies can be retained:

\footnotetext{
${ }^{11}$ For more details on the estimation of auto-spectral density functions and cross-spectral density functions, see Ftiti (2010).
} 
$\frac{\pi}{20}, \frac{4 \pi}{20}, \frac{7 \pi}{20}, \frac{10 \pi}{20}, \frac{13 \pi}{20}, \frac{16 \pi}{20}, \frac{19 \pi}{20}$. But we focus on only three frequencies in carrying out our analysis in the short, medium, and long terms. In practice, the shift from the frequency domain to the time domain is based on the following ratio: $\frac{2 \pi}{\lambda}$ where $\lambda$ is the frequency. The long-run coherence function for three years and three months is based on the frequency $\frac{\pi}{20}$, the middlerun of approximately one year refers to $\frac{4 \pi}{20}$, and the two months, short-run coherence is defined by the frequency $\frac{19 \pi}{20}$.

\subsubsection{Wavelet Approach: Theory and Estimation}

We choose a second frequency approach through wavelets useful for non-stationary time-series analysis. The wavelet approach enables investigating the relationship between two non-stationary time series through its continuously resized window properties. ${ }^{12}$

There are different wavelet groups that can be used in analyzing time series, such as discrete versus continuous and real versus complex wavelets. The continuous wavelet has been often used in previous studies (e.g., Aguiar-Conraria and Soares, 2011; Gallegati et al., 2014; Haven et al., 2012; Madaleno and Pinho, 2014; Rua and Nunes, 2012), as it is the most helpful with the time and scale resolution of the time-series decomposition and helps overcoming limitations of the other types of wavelet.

In our analysis, we choose the Morlet wavelet to obtain a better balance between time and scale resolutions. The Morlet wavelet was first introduced by Coupillaud et al. (1984), and can be presented as follows:

$$
\psi_{\Theta}(\mu)=\pi^{\frac{-1}{4}} e^{i w_{a} \mu} e^{\frac{-1}{2} \mu^{2}},
$$

where: $w_{0}$ and $\mu$ are defined as dimensionless frequency and time scales, respectively.

\footnotetext{
${ }^{12}$ In the case of low frequencies, the window width is wide, but narrow in the case of high frequencies. Consequently, a signal with a large window suggests coarse features, while a small window suggests fine features.
} 
For the Morlet wavelet, the central frequency $\left(w_{0}\right)$ is equal to six, which is considered a good choice to ensure a relevant balance between time and scale resolution (See Grinsted et al., 2004). For this central frequency, $w_{0}=6$, the Fourier period $\lambda_{w t}$ is almost equal to the scale $\left(\lambda_{w t}=\frac{w_{0}}{2 \pi}\right.$ scale $=\frac{6}{2 \pi}$ scale $\approx 1$ scale $)$. In addition, the relationship between the scale, $s$, and frequency, $f$, is given by $f \approx \frac{1}{s}$. The wavelet is drawn out in time by varying its scale, $s$, normalized to have unit energy, and defined as $s=\frac{\mu}{t}$. For the case of a discrete time series, $x_{n}\{n=1 \ldots, N\}$, of $N$ observations with a uniform time step ( $\left.\Phi t\right)$, the continuous wavelet transform is given by:

$$
W_{n}^{x}(s)=\sqrt{\frac{\Phi t}{s}} \sum_{m=1}^{N} X_{n} \Psi_{0}\left[(m-n) \frac{\Phi t}{s}\right]
$$

where $\Phi t$ is the time step.

The wavelet power spectrum for a time series, $x_{n}$, with $N$ observations is defined as $\left|W_{n}^{x}(\mathrm{~s})\right|^{2}$, and is defined as the local variance of time series, $x_{n}$. Thus, after defining the continuous wavelet transforms for each time-series analysis, in our case, $x_{t}$ (inflation) and $y_{t}(\mathrm{IU})$, we define the cross-wavelet transform. The measure of the cross-wavelet spectrum that captures the covariance between the two time series, $x(t)$ and $y(t)$, in the time-frequency space, $W_{x}$ and $W_{y}$, is defined as follows

$$
W_{n}^{x y}(s)=W_{n}^{x}(s) W_{n}^{y}(s)
$$

where $W_{n}^{x}(s)$ and $W_{n}^{y}(s)$ are the wavelet transforms for time series $x_{t}$ and $y_{t}$, respectively. The cross-wavelet power is defined by $\left|W_{n}^{x y}(s)\right|^{2}$ and is interpreted as the local covariance between two time series. ${ }^{13}$.

\footnotetext{
${ }^{13}$ More precisely, the cross-wavelet power between inflation and its uncertainty measures the similarity of the power in these series. The statistical significance level of this cross- wavelet power was defined by Torrence and Compo (1998),
} 
In the modeling of the causality relationship in the time domain, we define the phase difference, $\varphi_{x, y}$, between inflation, $x_{t}$, and its uncertainty, $y_{t}$. It is defined as the tool providing information about the delays in the oscillation between inflation, $x_{t}$, and its uncertainty, $y_{t}$ (Bloomfield et al., 2004). The phase difference depicts the relative position of the pseudo-cycle inflation and its uncertainty according to the following Equation (19):

$$
\varphi_{x, y}(s)=\tan ^{-1}\left(\frac{\mathcal{J}\left[w_{n}^{x y}(s)\right]}{\mathcal{R}\left[w_{n}^{x y}(s)\right]}\right)
$$

where, $\mathcal{J}$ and $\mathcal{R}$ denote the imaginary and real parts of the cross-wavelet, respectively.

To analyze the phase difference between inflation and its uncertainty, we note that it ranges between $[-\pi, \pi]$. If $\varphi_{x, y}(s)=0$, inflation and its uncertainty move together. This phase is analogous to positive covariance. When $\varphi_{x, y}(s) \in\left[-\frac{\pi}{2}, 0\right]$, inflation and its uncertainty are in phase, and inflation is leading. When $\varphi_{x, y}(s) \in\left[0, \frac{\pi}{2}\right]$, inflation and its uncertainty are in phase, and IU is leading. For $\varphi_{x, y}(s) \in\left[\frac{\pi}{2}, \pi\right]$, inflation and its uncertainty are in anti-phase, and inflation is leading. For $\varphi_{x, y}(s) \in\left[-\pi,-\frac{\pi}{2}\right]$, inflation and its uncertainty are in anti-phase, and IU is leading. ${ }^{14}$

Further to analyze the relationship between inflation and its uncertainty, we adopt wavelet coherence, as defined by Torrence and Webster (1999). This measure is associated with the coherence function defined in Equation (20) and the dynamic correlation in the conventional time series. However, the wavelet coherence function is superior to other measures, as it

\footnotetext{
${ }^{14}$ We note that in the coherence or cross-wavelet spectrum graphics, it is not easy to obtain a phase according to these different ranges. Therefore, the lead-lag relationship is reproduced through arrows pointed in different directions in the circular mean. This circular mean provides the signification of the phase lead-lag relationship. In order to determine the phase between two series, we must estimate the mean and confidence interval of the phase difference in line with Grinsted et al. (2004, pp. 4-5), who used the circular mean as defined by Zar (1999).
} 
identifies both the causality effect and the lead-lag phase phenomena between two time series. The wavelet coherence function is defined as

$$
\mathrm{R}_{\mathrm{n}}^{2}(\mathrm{~s})=\frac{\left|\varepsilon\left(\mathrm{s}^{-1} W_{n}^{x y}(\mathrm{~s})\right)\right|^{2}}{\varepsilon\left|\left(\mathrm{s}^{-1}\left|W_{n}^{x}(\mathrm{~s})\right|^{2}\right)\right| \cdot \varepsilon\left|\left(\mathrm{s}^{-1}\left|W_{n}^{y}(\mathrm{~s})\right|^{2}\right)\right|}
$$

where $\varepsilon$ is a smoothing operator. ${ }^{15}$ Since squared, our coherence function ranges from 0 to 1 , unlike a classical correlation measure, which would range from -1 to 1 .The statistical significance of the coherence function is estimated through a Monte Carlo method, in accordance with Torrence and Compo (1998) and Grinsted et al. (2004).

\section{Data and Empirical Analysis}

\subsection{Data and Preliminary Analysis}

The data include the consumer price indexes (CPI) for three developed regions, the US, the UK, and the Euro area, and two major emerging countries, China and South Africa. The CPI is required to compute the inflation rate from Equation 21, while the IU is computed using the stochastic volatility of inflation,

$$
\inf _{i, t}=100 * \operatorname{Ln}\left(\frac{C P I_{i, t}}{C P I_{i, t-1}}\right)
$$

where index $i$ represents the region.

Monthly data are obtained from Datastream and selected for each country, depending on the data availability: January 1999-March 2015, January 1988-March 2015, January 1950-March 2015, January 1960-December 2012, and January 1986-March 2015 for the Euro area, the UK, the US, South Africa, and China, respectively.

These countries show important changes in their conduct of monetary policies during these periods, including explicit versus implicit inflation targets and unconventional versus

\footnotetext{
${ }^{15}$ For more details, see Torrence and Webster (1998).
} 
conventional monetary policies. The US constitutes the best-documented case. There are at least four important phases known in its conduct of monetary policy: policy oriented toward unemployment in the 1970s, policy focused more on money after the 1979 oil shocks, policy focused on exchange rate and financial stability after the 1980s, and the unconventional monetary policies since $2008 .{ }^{16}$ On January 25, 2012, The Fed adopted an explicit inflation target of $2 \%$, but nevertheless continued to adopt a monetary policy with two main objectives, price stability and economic growth. Furthermore, the US monetary policy is characterized by emphasis on rules, but with a high degree of discretion, raising uncertainty about inflation. The UK is a major country to have adopted an explicit inflation target, which it did in 1992. In May 1997, the Bank of England acquired operational independence in setting its short-term interest rate. There are some similarities between the monetary histories of the US and the UK, in particular regarding their conduct of monetary policies and inflation targeting (see, e.g., Conrad and Karanasos, 2005). For the euro area, the European Central Bank adopted an implicit inflation target policy of around 2\%, although its policy covers several EU countries that did not apply an inflation target before 1999. However, ECB has only one stated objective, price stability. South Africa adopted an informal inflation target in 1990 and a formal one in 2000, ranging between $3 \%$ and 6\%. Finally, China's monetary policy aims to maintain currency stability and improve economic growth. China does not follow inflation targeting.

Accordingly, unlike previous studies, the inclusion of these countries with different target strategies, distinct degrees of independence, and heterogeneous monetary policies are intended to permit a useful comparative analysis of the inflation-IU relationship across countries.

We began by carrying out unit root tests finding that all inflation series are I(0). This result is consistent with the inflation rate dynamics in the studies mentioned in Figure $1 .{ }^{17}$ We report

\footnotetext{
${ }^{16}$ In the US, inflation increased due to the increase in defense spending in mid-1965 during the Vietnam war, after the first oil price shock in 1973, after the elimination of price and wage controls in 1974, and after the second oil shock during 1979-1980. See Bernanke and Mishkin (1992) for more details.

${ }^{17}$ We do not report the results of unit root tests to save space, but are available upon request.
} 
in Table 2 the main descriptive statistics.

Table 2. Descriptive Statistics of Inflation Rate and Normality Test

\begin{tabular}{|c||c|c|c|c|c|c|c||}
\hline & Mean (\%) & Min (\%) & Max (\%) & $\begin{array}{c}\text { Standard } \\
\text { deviation }\end{array}$ & Kurtosis & Skewness & Jarque-Bera test \\
\hline \hline UK & 0.220 & -0.618 & 2.338 & 0.238 & 22.16 & 2.463 & $(0.00)$ \\
\hline US & 0.290 & -1.735 & 1.846 & 0.343 & 5.555 & 0.171 & $(0.00)$ \\
\hline Euro area & 0.154 & -0.449 & 0.693 & 0.170 & 4.573 & -0.553 & $(0.00)$ \\
\hline China & -0.012 & -3.711 & 3.364 & 0.851 & 5.624 & -0.195 & $(0.00)$ \\
\hline South Africa & 0.672 & -0.742 & 4.118 & 0.553 & 7.013 & 1.212 & $(0.00)$ \\
\hline
\end{tabular}

Note: values in (.) denote p-values of the Jarque-Bera test.

Table 2, displays a lower level of inflation with a higher standard deviation for China than for the other regions. This volatility excess can be explained by the fact that, among these countries, only China does not pursue an objective of price stability. China has various other monetary objectives, including stability of currency value and promotion of economic growth, with use of several monetary instruments, including reserve requirement ratio, interest rate, rediscounting, lending, and open market operations. Kurtosis statistics are positive for all regions' inflation rates, with the highest value for the UK, suggesting fat tail behavior. The inflation rate distributions for the UK, the US, and South Africa are skewed to the right, while those for the Euro area and China are skewed to the left. This leptokurtic excess and asymmetry are inconsistent with normality, according to the Jarque-Bera test, and suggests that inflation might react differently after being shocked positively or negatively.

\subsection{Modeling the Dynamics of Inflation Uncertainty}

Figure 1 reports the dynamics of inflation rates, while Figure 2 plots the IU, based on the stochastic volatility measure described in subsection 3.1. We report in Table 3 the main descriptive statistics of the IU measure. 
Figure 1. Inflation rates for all sample countries

1(a) UK inflation rate

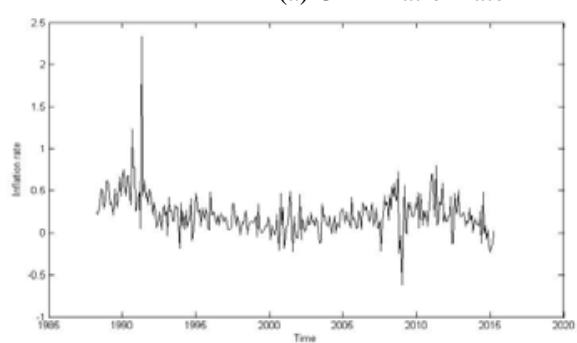

1(b) US inflation rate

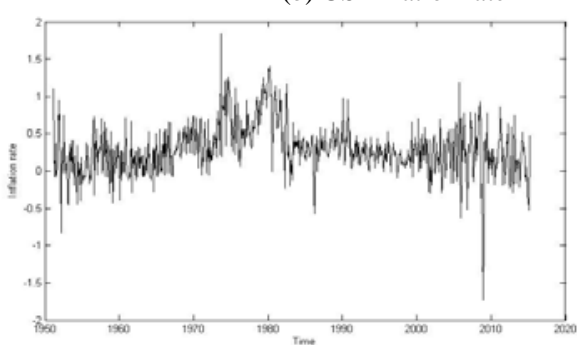

1(c) Euro area inflation rate

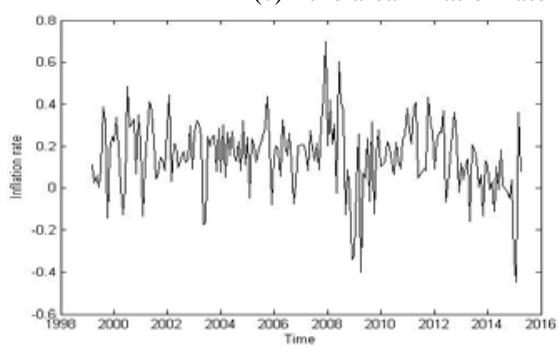

1(d) China inflation rate

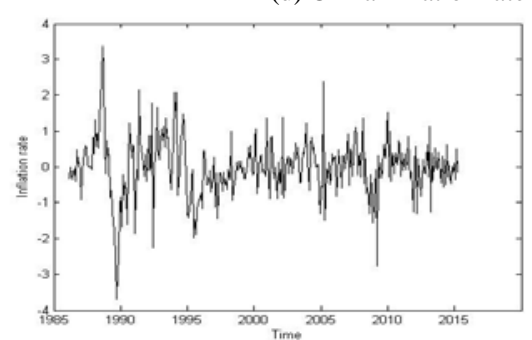

1(e) South Africa inflation rate

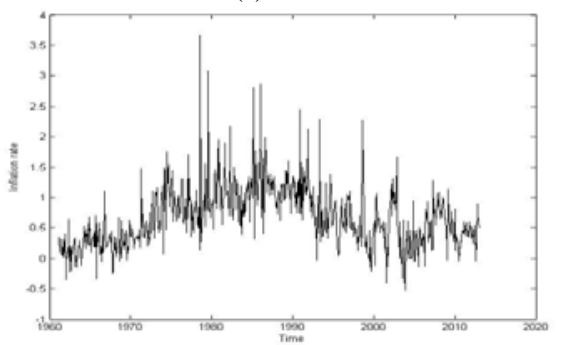

Figure 2. Inflation uncertainty for all sample countries

2(a) UK inflation uncertainty

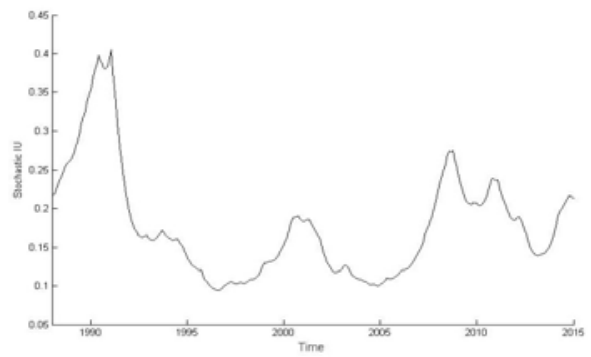

(b) US inflation uncertainty

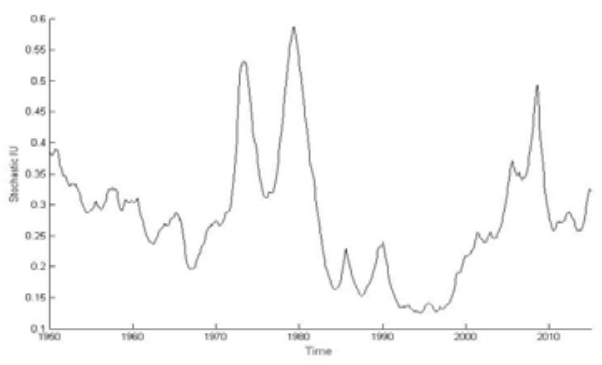

2(c) Euro area inflation uncertainty

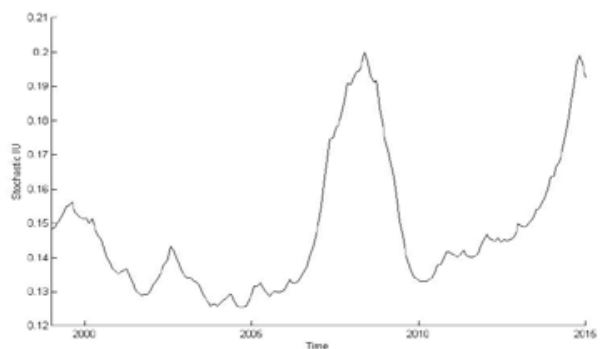

2(d) China inflation uncertainty

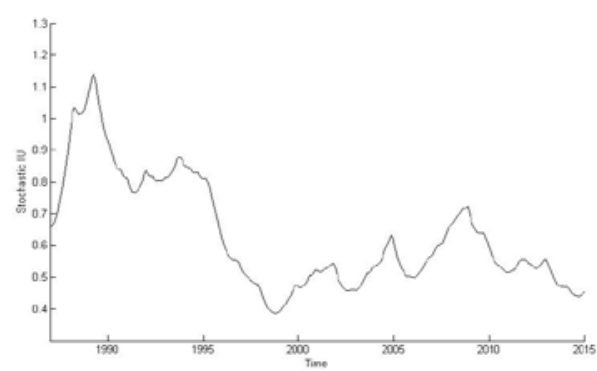

3(e) South Africa inflation uncertainty

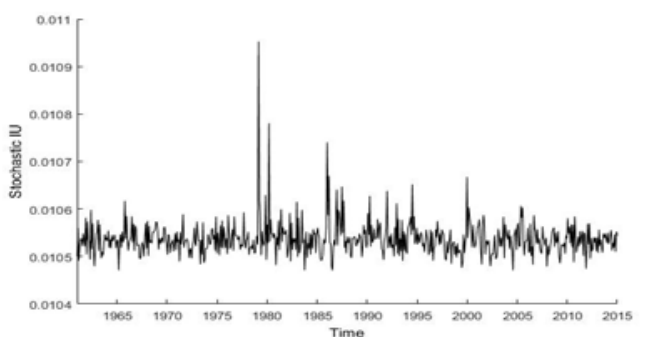


The following results are evident from those tables. China has the highest level of IU, even though it has the lowest inflation rate on average. In addition, China has the most volatile inflation and IU. This suggests that the absence of an explicit inflation target could be a source of an increase in uncertainty and volatility. Kurtosis statistics are positive for all IU, providing further evidence of fat tails. Further, all series of IU are skewed to the right, justifying the nonGaussian distribution of the conditional volatility of inflation. The Jarque-Bera test significantly rejects the normality for all series. Overall, these preliminary, indirect tests might suggest that IU could enter asymmetrically and nonlinearly with inflation, and thus exhibit a time-varying behavior.

Table 3. Summary Statistics Inflation Uncertainty Measure

\begin{tabular}{|c|c|c|c|c|c|c|c||}
\hline & Mean & Min & Max & $\begin{array}{c}\text { Standard } \\
\text { deviation }\end{array}$ & Kurtosis & Skewness & Jarque-Bera test \\
\hline \hline UK & 0.174 & 0.092 & 0.368 & 0.065 & 3.725 & 1.050 & $(0.000)$ \\
\hline US & 0.279 & 0.125 & 0.595 & 0.100 & 3.503 & 0.727 & $(0.000)$ \\
\hline Euro area & 0.153 & 0.126 & 0.217 & 0.025 & 3.125 & 1.113 & $(0.000)$ \\
\hline China & 0.617 & 0.419 & 0.898 & 0.126 & 2.104 & 0.575 & $(0.000)$ \\
\hline South Africa & 0.435 & 0.291 & 0.708 & 0.095 & 2.861 & 0.618 & $(0.000)$ \\
\hline
\end{tabular}

Note: value sin (.) refer to the p-values of the Jarque-Bera statistic test.

In order to investigate the inflation and IU properties directly and to specify their relationship, we first test the causality hypothesis between inflation and IU in a linear framework using the Granger Linear Causality Test. Next, we move to modeling this relationship in a nonlinear context with non-parametric econometric tests through a double time-frequency approach.

\subsection{Modeling the Relationship between Inflation and IU with parametric Tests}

The comparison of the inflation curve and IU dynamics in Figures 1 and 2 provides further evidence of similarities. For example, in the UK, when inflation increased at the beginning of the 1990s, the IU simultaneously experienced a peak, suggesting a positive relationship. But in 
the aftermath of the global financial crisis, the relationship seems to be the opposite, as inflation decreases but uncertainty increases, reflecting sign change in the relationship.

We also compute the non-conditional correlation matrix, apply the Granger causality test, and carry out some comparative analyses to analyze the inflation-IU relationship. See Table 4. The correlation is around 50\% for the UK and South Africa, but does not exceed 33\% for the U.S., as is consistent with Friedman's theory. However, the correlation appears null for China and negative for the Euro Area. The equality mean test significantly rejects the null hypothesis for the UK and South Africa. To better investigate this relationship, we conduct Granger causality tests and find evidence of bilateral causality relationships. However, given the rejection of the normal distribution, we have to analyze carefully the results of these parametric tests. To better understand the relationship between inflation and IU, we next apply two nonparametric approaches: evolutionary co-spectral analysis and the wavelet approach.

Table 4. Linkages between Inflation and Inflation Uncertainty

\begin{tabular}{|l|c|c|c|c|c|}
\hline & UK & US & Euro area & China & South Africa \\
\hline \hline Non-conditional correlation & 0.492 & 0.335 & -0.242 & 0.0174 & 0.485 \\
\hline \hline Granger causality test & \multicolumn{5}{l|}{} \\
\hline Inflation does not Granger cause inflation & $\begin{array}{c}44.750^{* * *} \\
(0.000)\end{array}$ & $\begin{array}{c}2.521^{*} \\
(0.081)\end{array}$ & $\begin{array}{c}3.653^{* *} \\
(0.027)\end{array}$ & $\begin{array}{c}3.389^{* *} \\
(0.035)\end{array}$ & $\begin{array}{c}77.497^{* * *} \\
(0.000)\end{array}$ \\
\hline uncertainty & & & & 0.619 & $29.858^{* * *}$ \\
& & & $(0.000)$ \\
\hline Inflation uncertainty does not Granger & $\begin{array}{c}18.666^{* * *} \\
(0.000)\end{array}$ & $\begin{array}{c}9.121^{* * *} \\
(0.000)\end{array}$ & $\begin{array}{c}2.555^{*} \\
(0.080)\end{array}$ & $(0.538)$ & 0.000 \\
\hline \hline Eause inflation & 0.001 & 0.398 & 0.980 & 0.800 & 0 \\
\hline
\end{tabular}

Note: values in (.) denote the p-values for different tests. ***, **, and * indicate significance levels of $1 \%, 5 \%$, and $10 \%$, respectively.

\subsection{Modeling the Relationship between Inflation and IU with Nonparametric Tests}

\subsubsection{Evolutionary Co-Spectral Analysis}

Figures 3, 4, and 5 report the dynamic interaction between inflation and IU for the long, medium, and short terms, respectively, where long run is defined to be more than three years, medium run one year, and short run three months. Overall, those figures point to several conclusions. The relationship between inflation and uncertainty exhibits a significant time variation, which confirms the dynamic linkages between inflation and IU, as noted by previous 
related empirical studies. Our specification captures the most important stylized facts associated with inflation increases. Indeed, the coherence function reaches high levels during periods of crises and shocks, including the oil shocks, dot-com bubble, and the recent global financial crisis, while reflecting the peak of uncertainty during market downturns.

While considering the different patterns, we note that the long-run coherence function is relatively smooth for the UK and the Euro area, but is more volatile for the US, China, and South Africa. Lower average correlation is observed in the case of the UK and higher for China. More specifically, for the UK, we observe a breakdown of the dynamic interdependence between inflation and IU in 1992, which is when the inflation targeting policy was adopted. This dramatic decrease is explained by the fundamentals of the inflation targeting policy, which is characterized by a high degree of commitment and transparency, and a low level of inflation uncertainty. Since 1992, the correlation level has remained lower and has only increased at the beginning of 2000, reflecting the increased housing prices, leading to a high interest rate and high taxes on house purchases.

Regarding the US, the highest levels of the coherence function reflect the price level during certain periods of the 1960s (Vietnam War), of 1973 and 1979 (oil shocks), of the end of the 1980s, of 2000 (dot-com bubble), of 2007-2008 (subprime and financial crisis), and of 2010. In the Euro area, the coherence function reached high levels after the subprime crisis and during the sovereign debt crisis. In China, the relationship reached high levels during the dot-com bubble, reflecting the absence of an explicit inflation target. In South Africa, the dependence between inflation and IU was low and stable from 1960 to the end of the 1990s. At the beginning of 2000, the dynamic interaction between the series grew and reached high levels.

Overall, the nonparametric analysis of the relationship between inflation and IU provides the following results. It identifies the linkage dynamics by sub-period, determines the association with monetary regime policy, captures the different stylized facts, and yields an 
analysis of this relationship. It also shows that inflation-IU relationship might significantly vary across countries, reflecting the effects of central bank policies, their targeting rules, and degrees of interdependence.

Figure 3. Long-run coherence function between inflation and IU

3(a) UK

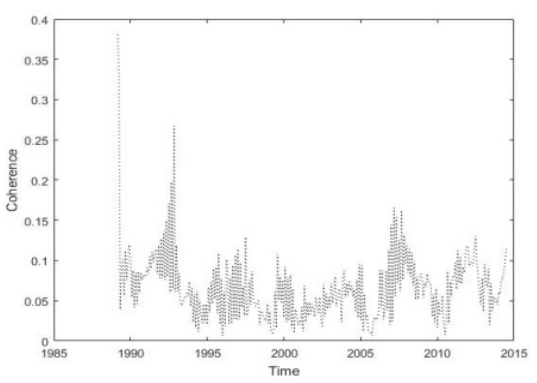

3(c) Euro area

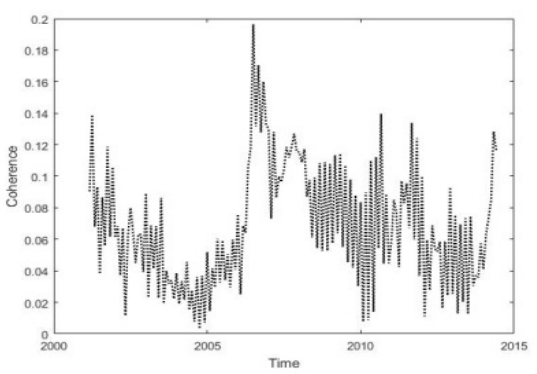

3(b) US

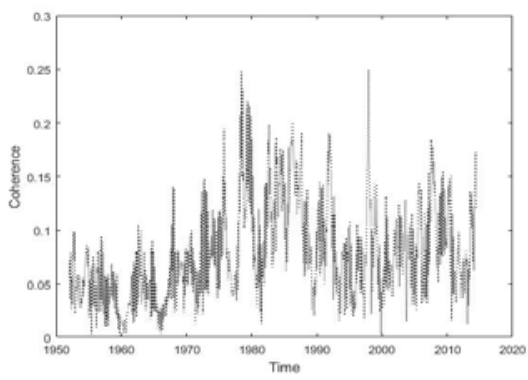

3(d) China

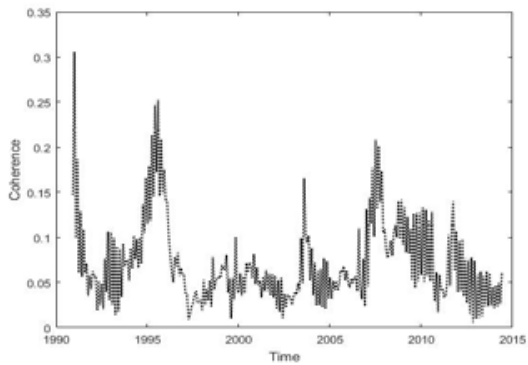

3(e) South Africa

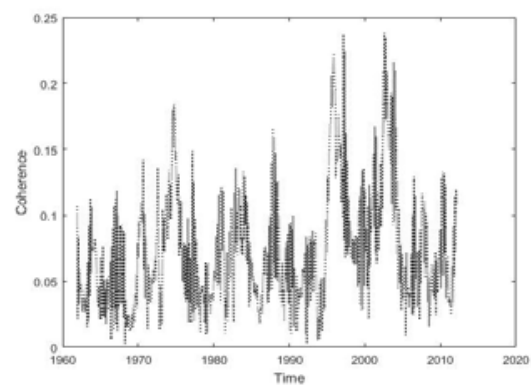

Regarding the pattern of the medium and short runs, the interdependence between inflation and IU is significantly more important in the short and medium terms than in the long term. On average the long-run coherence function did not exceed $15 \%$ for some countries, but the medium- and short-run coherence functions are significantly higher, at $60 \%$ and $70 \%$, 
respectively (Figures 4 and 5). Finally, while the estimate of the short-run coherence function enables capturing the intensity of interaction between inflation and uncertainty, it also becomes clear that the interaction dynamic is very high during periods of turmoil (around $70 \%-80 \%$ ) and relatively low during the stable periods (30-50\%).

In summary, the evolutionary co-spectral analysis points to a significant relationship between inflation and IU, more pronounced in the medium and short runs than in the long run. This relationship exhibits significant time variation associated with the market state. The intensity of the relationship increases significantly during periods of turmoil (70\%-80\%), while it is lower (30-50\%) during calm periods. This finding confirms the usefulness of our spectral approach, which demonstrates that the uncertainty effects could vary across horizons. Investigating this relationship for different horizons can assist policymakers to limit the uncertainty effects for each horizon. 
Figure 4. Medium-run coherence function between inflation and IU

4(a) UK

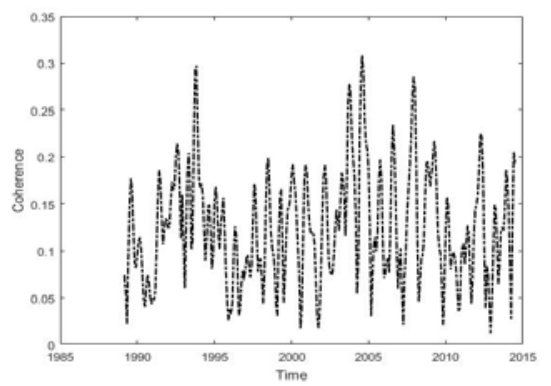

4(c) Euro area

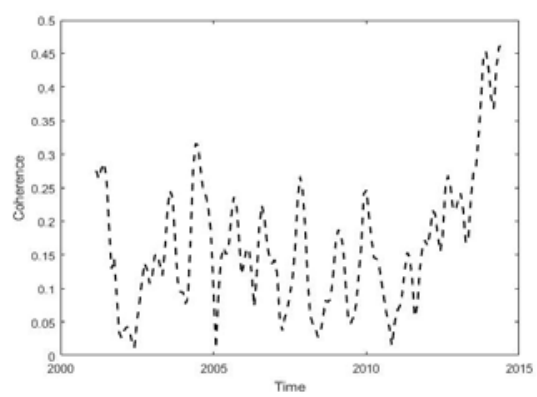

4(b) US

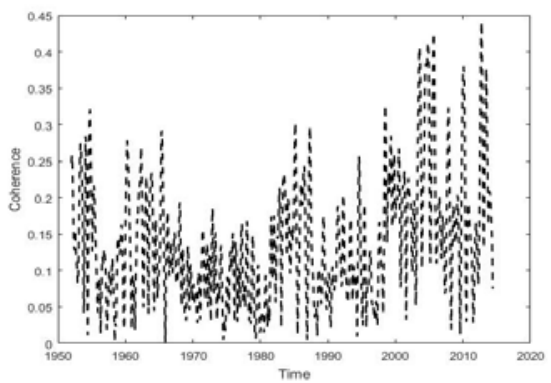

4(d) China

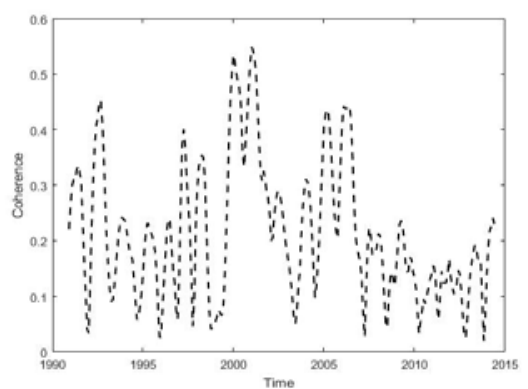

4(e) South Africa

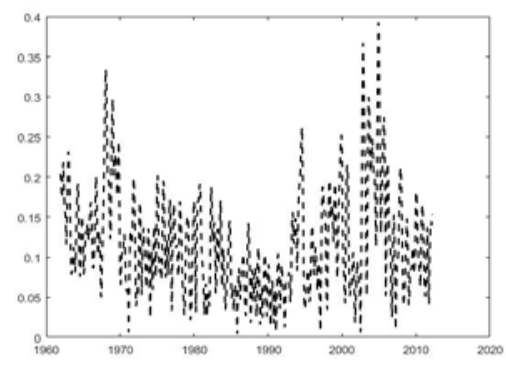

This evolutionary co-spectral analysis contributes to the related literature by clarifying this relationship. Not only does it enable identifying periods with high and low interactions, but also permits measuring the effects of policymaker actions, such as explicit inflation targeting and the degree of central bank independence. However, the evolutionary co-spectral analysis does not provide the statistical significance of these relationships, only the estimated magnitude of interdependence. To conduct the statistical significance tests, the wavelet approach is used to complete this analysis and fill the gap. 
Figure 5. Short-run coherence function between inflation and uncertainty

5(a) UK

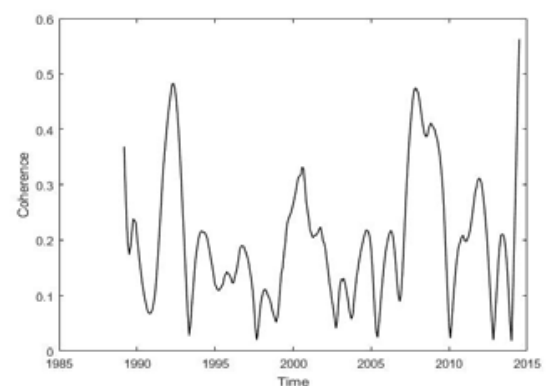

5(c) Euro area

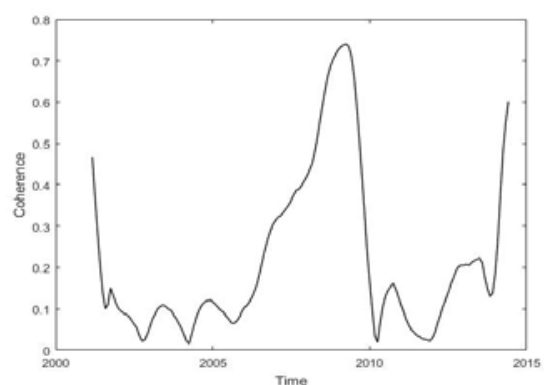

5(b) US

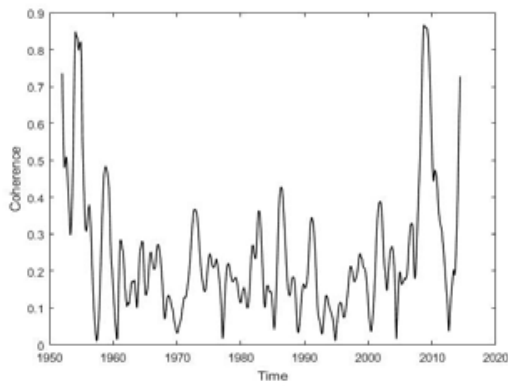

5(d) China

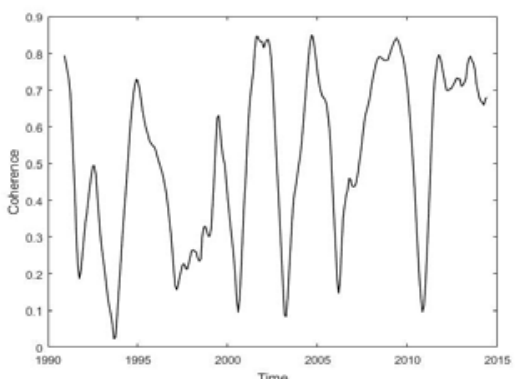

5(e) South Africa

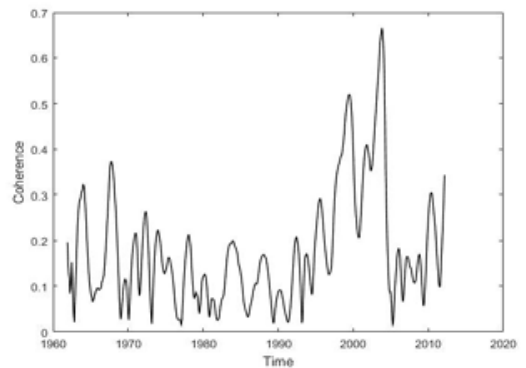

\subsubsection{Wavelet Analysis}

In Figure 6, we report the results of the coherence wavelet function between inflation and IU. The vertical and horizontal axes refer to the time and frequency dimensions, respectively, while the color depicts the level of interdependence, varying between zero and one. A lower interdependence is indicated by dark blue, and a higher correlation is represented by dark red. The left vertical color axis provides more precise information about the level of interdependence. The main contribution of the wavelet approach is to provide the sign of 
causality between inflation and IU. Arrows pointing to the right imply that variables are in a pro-cyclical phase. More precisely, when arrows point right and up, they imply that IU is lagging, while when they point right and down, they imply that IU is leading. When the arrows point to the left, the series are counter-cyclical. More precisely, when they point left and down, IU is lagging, and when they point left and up, IU is leading.

The wavelet coherence function analysis provides two clear conclusions. First, we confirm the analysis provided by the evolutionary co-spectral analysis in terms of significant timevariation of co-movements between inflation and IU. The co-movements vary by country and are more pronounced in the short term than the medium and long terms. Figure 6(a) displays a high interdependence between the British inflation rate and its uncertainty at the beginning of $90 \mathrm{~s}$ in the short and medium horizons (0-16 scales). For the short horizon (0-4 scales), the interdependence between UK inflation and IU is high in 1997, in 2000-2001 (internet bubble), and in 2008-2009 (subprime crisis). For the medium term, the relationship between inflation and IU is important during two episodes: the period of the "great moderation" (1990s) and during the subprime crisis (2008-2009). In the long term, the relationship between inflation and IU is observed only during the 1990s.

For the US, dependence is observed in the short, medium and long horizons during the periods of turmoil, including in the end of 60 s, in the 70s and 80s, and in the beginning of 90s in the short and medium run. Since the latter period, the interdependence is observed only in the short and medium horizons, such as during the subprime crisis. For the Euro area, the relationship is observed in the short term (0-8 scales) during the periods 2001-2002 (the internet bubble), 2007-2008 (the subprime crisis), and 2011-2012 (European sovereign debt problem). Furthermore, a long run relationship is observed during the period 2004-2008. Finally, a similar behavior is observed for South Africa, where the interdependence is more pronounced in periods of turmoil. In particular, the relationship between inflation and IU is higher during the 
1970s in the short and medium terms (0-16 scales). This behavior has been reproduced during the period 1985-1988 for the short and medium terms. A high interdependence is also observed during the beginning of 2000 in the medium horizon.

\section{Figure 6. Coherence between inflation rate and the inflation uncertainty}

12(a) UK

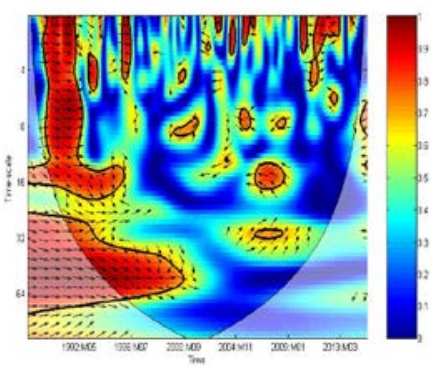

12(c) Euro area

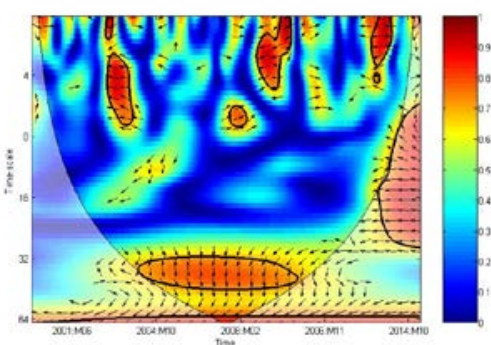

12(b) US

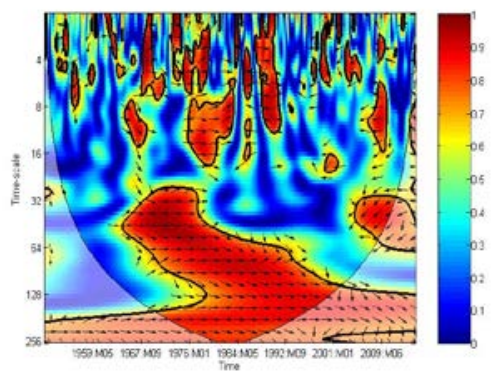

12(d) China

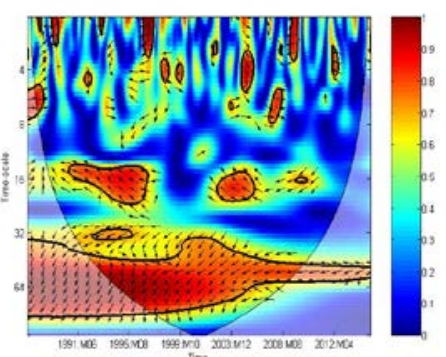

12(e) South Africa

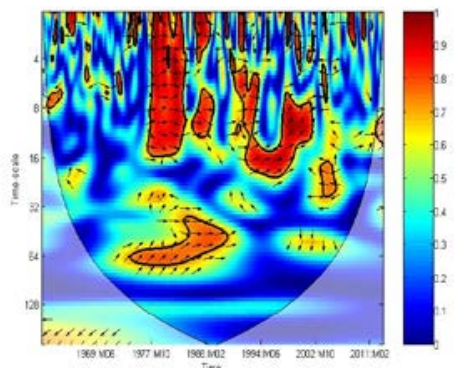

The wavelet analysis confirms the findings of the evolutionary co-spectral approach and shows a significant relationship that is more pronounced in the medium and short terms than in the long term. The intensity of the relationship increases significantly during periods of turmoil, while the long-term relationship is observed only in periods of crisis. This finding is consistent with Evans (1991) decomposition of inflation uncertainty into short-run and long-run. He suggests that agents' temporal decisions are more likely sensitive to the conditional variance of short-run movements in inflation. However, intertemporal decisions are more likely dependent 
on changes in the conditional variance of long-term inflation. Indeed, we have identified a high relationship between inflation and IU in the short term. This relationship can be explained by the agent's temporal decisions during a stable period, such as their inflation forecasting regarding the state of economy. We also found a long-run relationship during a period of crisis that is explained by intertemporal decisions, such as change of monetary policy in response to crises or structural change to overcome some economic instability. See, e.g., Caporale and Kontonikas (2009).

In addition, the wavelet approach produces different directions of the arrows, suggesting that the relationship exists but alternates between positive and negative, according to the economic state and monetary policy. In some cases, the arrows point left, implying that inflation and uncertainty may be counter-cyclical, such as in the Euro area during periods 2001-2002, 2007-2008, and 2011-2012 for the short run; the US 2008-2009 for the medium run; and China during 1990-1995 for the long run. In other cases, the arrows point right, implying a procyclical relationship, such as in the UK during 1990-1995 for the short and medium run, in 1997 for the short run, and during 2009-2012 in the short run. For the US, the arrows point right during all the periods of high interdependency described above, except the period of subprime crisis, where arrows point to the left. For South Africa, the arrows point right for all periods in the scales between 0 and 32. These findings confirm the stylized facts observed in Figure 1, when we highlight similar movements in the same direction between these series in some cases and in opposite directions in others. In addition, our results show that causality varies across frequencies. In some cases, inflation is lagging and in others, it is leading.

For all studied countries, we observe that for the short-term frequency, the arrows are pointed left down, right up, or right down, implying counter-cyclical or pro-cyclical relationships, with IU leading or lagging. These findings support the theoretical hypotheses summarized in table 1 . In short, the relationship between inflation and IU might be positive or 
negative and the causality alternates across frequencies and countries. For example, for the UK in the short run, the arrow is pointing right and down during stable periods. In the beginning of the 1990, the arrow points right, implying a pro-cyclical positive relationship between inflation and IU, with IU leading for short and medium terms. During the subprime crisis, the arrows are pointed right and down for medium frequency and right and up for long term frequency, implying a pro-cyclical positive relationship between inflation and IU. The causality alternates between horizons. This result supports the Friedman-Ball and Cukierman and Meltzer (1986) hypotheses. The change of the causality in the long-term frequency after the subprime crisis might be explained by the nonconventional policy adopted, having objective to reduce uncertainty and to ensure more stability in the economy. As this period is characterized by a high degree of economic uncertainty, the IU could not continue to be predictive of the inflation.

The pattern of the relationship between inflation and IU is different for the US. In the short term, the arrows are pointed right and up from the 1950s to the end of the 1990s. This result reflects a procyclical relationship with inflation causing the IU. Since the 2000s, the relationship is only observed in periods of turmoil (2001, and the subprime crisis). During these periods, the arrows are pointed left and down, implying that the relationship is countercyclical, with IU causing inflation. For the Euro area and China, the arrows usually point left, and the causality alternates between IU lagging and leading. For South Africa, the arrows are pointed right and up for all time-scales, implying procyclical relationship, with inflation causing IU.

\subsection{Robustness Tests}

The robustness check concerns the coherence determined through the wavelet approach. In our analysis, we rely on the theoretical distribution defined by Torrence and Compo (1998), as presented in Equation (12). This distribution has been criticized by Liu et al. (2007) and Veleda et al. (2012) in terms of low-frequency oscillations, leading to some ambiguity in the wavelet power spectrum estimation. For robustness confirmation and to avoid bias, we re-estimate the 
cross-wavelet spectrum, as defined by Ng and Chan (2012). The results show significant similarities using two different estimation methods. ${ }^{18}$ This confirms the robustness of our findings and conclusions.

\section{Conclusion}

The measure of IU is a crucial topic for both policymakers and economic agents. For policymakers, a relevant measure of uncertainty leads to the adoption of appropriate monetary policy actions, which is more active in the case of high uncertainty and less so otherwise. For economic agents, higher uncertainty leads to more frequent negotiations of nominal contracts. This study analyzes the relationship between inflation and IU for five major countries and regions (the US, the UK, the Euro area, China, and South Africa). The topic is investigated during crises and downturn periods in the context of economies with explicit versus implicit inflation targets, conventional versus unconventional monetary policy, and independent versus dependent monetary policy.

Our paper estimates the IU using the stochastic volatility model. We also propose a novel econometric specification for the inflation-IU relationship. Our findings show different relevant results. First, there is a significant relationship between inflation and uncertainty that exhibits time-variation and changes across frequencies and time. Indeed, the linkages are more significant in the short term than in the long term. In addition, this relationship seems to increase during periods of crises and downturns. Second, this relationship alternates between being positive for stable periods, where IU is lagging — thereby confirming the Friedman theoryand negative during crises. Finally, the significant differences between the countries under consideration highlight the effect of monetary regimes on uncertainty, and could be helpful in selecting the appropriate and timely monetary policy to limit uncertainty effects.

Our results contribute to the literature in various ways. The results support all the

\footnotetext{
${ }^{18}$ To save place, we have not reported the graphs of these results. They are available upon author request.
} 
contradictory results in the previous literature, in particular by resolving the complexity and ambiguity noted in previous related results on the inflation-IU relationship. More specifically, our results highlight that the relationship between inflation and IU can be positive (the BallFriedman hypothesis) or negative (the Holland hypothesis), depending on whether the economic environment is stable or turbulent. Our results show the relationship across frequency and time. We provide insights relevant to reducing or minimizing the marginal effect of inflation on IU. In different contexts—such as calm or turbulent periods and price stability monetary objectives — this relationship depends on the monetary policy under consideration. We show that uncertainty is lower in the case of a price stability objective.

Previous studies have found varying and seemingly conflicting relationships between inflation and IU. Our more general approach nests the prior results into a unified framework, making clear the circumstances producing prior appearances of ambiguity of the relationship and identifying the causation in each case.

\section{References}

Aguiar-Conraria, L., Azevedo , N., Soares, M. J., 2008. Using wavelets to decompose the timefrequency effects of monetary policy. Physica A: Statistical Mechanics and its Applications. 387. 2863-2878.

Allégret, J.P., Essaadi, E., 2011. Business cycles synchronization in East Asian economy: Evidences from time-varying coherence study. Economic Modelling. 28, 351-365.

Allen, M.R., Smith, L.A., 1996. Monte Carlo SSA: Detecting irregular oscillations in the presence of colored noise, J. Climate. 9, 3373-3404.

Artis, M.J., Bladen-Hovell, R., Nachane, D.M., 1992. Instability of the velocity of money: A New approach based on the evolutionary spectrum. CEPR Discussion Paper, $\mathrm{n}^{\circ} 735$.

Baillie, R.T., Chung, C., Tieslau, M.A., 1996. Analysing inflation by the fractionally integrated 
ARFIMA-GARCH model. J. Appl. Econometrics. 11, 23-40.

Ball, L., 1992. Why does high inflation raise inflation-uncertainty? J. Monetary Economics. 29, 371-388.

Barnett, W. A., Chen, G. (2015). Bifurcation of macroeconometric models and robustness of dynamical inferences. Foundations and Trends in Econometrics. 8 (1-2), 1-144.

Barnett, W. A., Serletis, A. and Serletis, D. (2015). "Nonlinear And Complex Dynamics In Economics," Macroeconomic Dynamics, Cambridge University Press, vol. 19(08), pages 17491779.

Barnett, W. A., Gallant, A. R., Hinich, J. A., Jungeilges, D. T., Kaplan, D. T., and Jensen, M. J., 1997. A single-blind controlled competition among tests for nonlinearity and chaos, Journal of Econometrics. 82, 157-192.

Barro, R.J., Gordon, D.B., 1983. Rules, discretion and reputation in a model of monetary policy. J. Monetary Economics. 12, 101-121.

Ben Nasr, A., Balcilar, M., Ajmi, A.N., Aye, G.C., Gupta, R., Eyden, R., 2015. Causality between inflation and inflation uncertainty in South Africa: Evidence from a Markov-switching vector autoregressive model. Emerg. Markets Rev. 24, 46-68.

Bernanke, B.S., Mishkin, F.S., 1992. Central bank behavior and the strategy of monetary policy: Observations from six industrialized countries. NBER Working Paper No. 4082.

Berument, H., Yalcin, Y., Yildirim, J., 2009. The effect of inflation uncertainty on inflation: Stochastic volatility in mean model within a dynamic framework. Economic Modelling. 26, 1201-1207.

Bloomfield, D.S., McAteer, R.T.J., Lites, B.W., Judge, P.G., Mathioudakis, M., Keenan, F.P., 2004. Wavelet phase coherence analysis: Application to a quiet-sun magnetic element. The Astrophysical J. 617, 623-632. 
Bollerslev, T., Wooldridge, J., 1992. Quasi-maximum likelihood estimation and inference in dynamic models with time-varying covariance. Econometric Rev. 11, 143-172.

Bouoiyour, J. and Selmi, R. 2014. Nonlinearities and the nexus between inflation and inflation uncertainty in Egypt: New evidence from wavelet transform framework. MPRA, No. 55721.

Canzoneri, M., 1985. Monetary policy games and the role of private information. American Economic Review. 75. 1056-1070.

Caporale, G.M., Kontonikas, A., 2009. The Euro and inflation uncertainty in the European Monetary Union. J. International Money and Finance 28(6): 954-971.

Carnero, M. A., Pena, D., and Ruiz, E. 2004. Persistence and kurtosis in GARCH and stochastic volatility models. Journal of Financial Econometrics 2:3, 19-342.

Chan, J. and Grant, A. 2016. Modeling energy price dynamics: GARCH versus stochastic volatility. Energy Economics 54, 182-189.

Chan J.C.C., Hsiao, C.Y.L., 2014. Estimation of stochastic volatility models with heavy tails and serial dependence, in: Jeliazkov, I. Yang, X.-S. (Eds.), Bayesian Inference in the Social Sciences. John Wiley \& Sons, Hoboken.

Chan, J.C.C., 2013. Moving average stochastic volatility models with application to inflation forecast. J. Econometrics. 176 (2), 162-172.

Chan, J.C.C., 2015. The stochastic volatility in mean model with time-varying parameters: An application to inflation modeling. J. Bus. Economic Statistics. Forthcoming.

Chang, K., 2012.The impacts of regime-switching structures and fat-tailed characteristics on the relationship between inflation and inflation uncertainty. J. Macroeconomics 34,523-536.

Conrad, C., Karanasos, M., 2005. On the inflation-uncertainty hypothesis in the USA, Japan and the UK: A dual long memory approach. Jpn. World Economy. 17, 327-343. 
Creal, D.D., Wu, J.C., 2014. Interest rate uncertainty and economic fluctuations, Working paper n¹4-32 4. The University of Chicago Booth School of Business.

Cukierman, A., Meltzer, A.H., 1986. A theory of ambiguity, credibility, and inflation under discretion and asymmetric information. Econometrica. 54, 1099-1128.

Cukierman, A., 1992. Central Bank Strategy, Credibility, and Independence: Theory and evidence, MIT Press, Cambridge.

Goupillaud, P., Grossman, A., Morlet, J., 1984. Cycle-octave and related transforms in seismic signal analysis. Geoexplor. 23, 85-102.

Darné, O., 2004. Les méthodes et logiciels de désaisonnalisation des séries économiques: une revue de la littérature, Journal de la Revue Française de la Statistiques. 145, 79-102.

Emery, K.M., 1993. Inflation and its variability: An alternative specification. Appl. Economics 25, 43-46.

Evans, M., 1991. Discovering the link between inflation rates and inflation uncertainty. J. Money, Credit Bank. 23 (2), 169-184.

Evans, M., Wachtel, P. 1993. Inflation regimes and the sources of inflation uncertainty. J. Money Credit Bank. 25, 475-511.

Ferreira, D. and Palma, A. 2016. Inflation and inflation uncertainty in Latin America: A timevarying stochastic volatility in mean approach. Journal of Economic Studies 44(4) 506-517.

Fischer, S., Modigliani, F., 1978. Toward and understanding of the real effects and costs of inflation. Weltwirtschaftliches Archiv. 114, 810-833.

Friedman, M., 1977. Nobel lecture: Inflation and unemployment. J. Political Economy. 85, 451-472. 
Ftiti, Z., 2010. The macroeconomic performance of the inflation targeting policy: An approach based on the evolutionary co-spectral analysis. Economic Modelling. 27, 468-476.

Ftiti, Z. and Jawadi, F. (2018), Forecasting inflation uncertainty in the United States and Euro Area. Computational Economics, forthcoming.

Gallegati, M., Ramsey, J.B., Semmler, W., 2014. Interest rate spreads and output: A time scale decomposition analysis using wavelets. Computational Statistics and Data Analysis. 76, 283290.

Gencay, R., Selçuk, F., and Witcher, B. (2001), An Introduction to Wavelets and Other Filtering Methods in Finance and Economics, Academic Press.

Golub, J., 1994. Does inflation uncertainty increases with inflation. Federal Reserve Bank of Kansas City.Third Quarter, 28-38.

Gourieroux, C., Sufana, R., 2010. Derivative pricing with Wishart multivariate stochastic volatility. J. Bus. Econom. Statist. 28, 438-451.

Grier, K.B., Perry, M.J., 1998. On inflation and inflation uncertainty in the G7 countries. J. International Money and Finance. 17, 671-689.

Grinsted, A., Moore, J., Jevrejeva, S., 2004.Application of the cross wavelet transform and wavelet coherence to geophysical time series. Nonlinear Processes in Geophysics.11,561-566.

Haven, E., Liu, X., Shen, L., 2012. De-noising option prices with the wavelet method. European J. Operational Res. 222, 104-112.

Holland, S., 1993. Comment on inflation regimes and the sources of inflation uncertainty. J. Money Credit Bank. 25, 514-520.

Holland, S., 1995. Inflation and uncertainty: Tests for temporal ordering. J. Money Credit Bank. 27, 827-837. 
Johnson, D.R., 2002. The effect of inflation targeting on the behavior of expected inflation: Evidence from an 11 country panel. J. Monetary Economics. 49, 1521-1538.

Kontonikas, A., 2005. Inflation and inflation uncertainty in the United Kingdom, evidence from GARCH modelling, Economic Modelling. 21, 525-543.

Koopman, S.J., Mallee, M.I., Van der Wel, M., 2010. Analyzing the term structure of interest rates using the dynamic Nelson-Siegel model with time-varying parameters. J. Bus. Economic Statistics. 329-343.

Liu, Y., Liang, X.S., Weisberg, R.H., 2007. Rectification of the bias in the wavelet power spectrum. J. Atmospheric Ocean. Technology. 24, 2093-2102.

Logue, D., Willett, T., 1976. A note on the relation between the rate and variability of inflation. Economica. 43, pp. 151-158.

Madaleno, M., Pinho, C., 2014. Wavelet dynamics for oil-stock world interactions. Energy Economics. 45, 120-133.

Mallick, S, and Sousa, R. (2013), “Commodity prices, inflationary pressures, and monetary policy: Evidence from BRICS economies”, Open Economies Review, 24(4), 677-694.

Mallick, S. and Mohsin, M. (2016) Macroeconomic Effects of Inflationary Shocks with Durable and Non-Durable Consumption, Open Economies Review, 27(5): 895-921.

Neanidis, K.C., and Savva, C.S., 2011. Nominal uncertainty and inflation: The role of European Union membership. Economic letters. 112, 26-30.

Nelson, D. B., 1991. Conditional heteroskedasticity in asset returns: A new approach. Econometrica. 59, 347-370.

Ng, E.K., Chan, J.C., 2012. Geophysical applications of partial wavelet coherence and multiple wavelet coherence. J. Atmospheric Ocean. Technology. 29, 1845-1853. 
Pourgerami A, Maskus KE (1987) The effects of inflation on the predictability of price changes in Latin America: Some estimates and policy implications. World Development 15(2): 287290.

Priestley, M.B., 1965. Evolutionary spectra for non-stationary processes. J. R. Statistical Society. Series B 27, 204-237.

Priestley, M.B., 1966. Design relations for non-stationary processes. J. R. Statistical Society. Series B 28, 228-240.

Priestley, M.B., 1988. Non-Linear and Non-Stationary Time Series Analysis, Academic Press, London.

Priestley, M.B., Tong, H., 1973. On the analysis of bi-variate non-stationary processes. J. R. Statistical Society. Series B 35, 135-166.

Rua, A., Nunes, L.C., 2012. A wavelet-based assessment of market risk: The emerging markets case. Q. Rev. Economics Finance. 52, 84-92.

Rossi, B., Sekhposyan, T. and Soupre, M. 2016. "Understanding the Sources of Macroeconomic Uncertainty," Working Papers 920, Barcelona Graduate School of Economics.

Serletis, A. and Rahman, S., 2009a. The output effects of money growth uncertainty: Evidence from a multivariate GARCH-in-Mean VAR. Open Economies Review. 20, 607-630.

Serletis, A. and Rahman, S., 2009b. On the output effects of monetary variability. Open Economies Review 26. 225-236.

Serletis, A. and Shahmoradi, A., 2006. Velocity and variability of money growth: Evidence from a VARMA, GARCH-M model. Macroeconomic Dynamics. 10, 652-666.

Serletis, A. and Xu, L., 2017. Money supply volatility and the macroeconomy, University of Calgary, working paper. 
Torrence, C., Compo, G.P., 1998. A practical guide to wavelet analysis, Bull. American Meteorological Society. 79, 605-618.

Torrence, C., Webster, P., 1999. Interdecadal changes in the ENSO-monsoon system. J. Climate. 12, 2679-2690.

Ungar, M., Zilberfarb, B., 1993. Inflation and its unpredictability-theory and empirical evi-dence. J. Money, Credit Bank. 25, 709-720.

Van Bellegem, S., 2013. Locally stationary volatility modelling, in: Bauwens, L., Hafner, C., Laurent, S. (Eds.), Handbook in Financial Engineering and Econometrics: Volatility models and their applications. Wiley, New York, chapter 10.

Van Bellegem, S., Von Sachs, R., 2008. Locally adaptive estimation of evolutionary wavelet spectra. J. Statistics. 36, 1879-1924.

Veleda, D., Montagne, R., Araujo, M., 2012. Cross-wavelet bias corrected by normalizing scales. J. Atmospheric Ocean. Technology. 29, 1401-1408.

Zapodeanu, D., Cociuba, M-L., Sorina, P., 2014. Inflation uncertainty and inflation in the case of Romania, Czech Republic, Hungary, Poland and Turkey. Procedia Economics and Finance. $15,1225-1234$.

Zar, J.H., 1999. Biostatistical Analysis, fourth ed. Prentice Hill. 